\title{
Temperature dependence of the spectral band shape of CdSe nanodots and nanorods
}

\author{
J. I. Climente, J. Planelles, ${ }^{*}$ and F. Rajadell \\ Departament de Química Física i Analítica, Universitat Jaume I, P.O. Box 224, E-12080 Castelló, Spain
}

(Received 2 August 2009; revised manuscript received 9 October 2009; published 12 November 2009)

\begin{abstract}
We study theoretically the effect of thermal population on the emission spectrum of single CdSe nanocrystals. Quantum confinement leads to nonsimple emission band shapes, which have different characteristics for excitons, biexcitons, positive, and negative trions. These effects are particularly pronounced in nanorods. The maximum of the emission band is not necessarily centered at the fundamental transition energy.
\end{abstract}

DOI: $10.1103 /$ PhysRevB.80.205312

PACS number(s): 73.21.La, 71.35.Pq, 71.35.Cc, 78.67.Hc

\section{INTRODUCTION}

Colloidal CdSe nanocrystals are semiconductor quantum dots with excellent prospects as room-temperature fluorophores for optoelectronics and biolabeling applications. ${ }^{1,2}$ Their light-emission properties rely ultimately on the details of the electronic band structure. Theoretical descriptions of the electronic structure based on effective mass or atomistic models have successfully interpreted many phenomena observed in low-temperature experiments. ${ }^{3,4}$ The next step is to understand the influence of temperature $(T)$ on the emission spectrum, as nanocrystal devices are expected to operate at room temperature. A number of recent experiments focused on the $T$ dependence of the band gap of spherical CdSe nanocrystals or nanodots (NDs). ${ }^{5-8}$ It was shown that most of the variation with increasing $T$ comes from the bulklike shrinkening of the gap with an additional and comparatively small contribution coming from changes in the confined electronic states. The spectral line shape of single NDs was also investigated. ${ }^{4}$ At low $T$ it is often determined by radiative processes involving surface states. Surface-state resonances are usually weakly luminescent and redshifted from those of the volume states. Still, they lower the symmetry of the nanocrystal, hence enabling otherwise forbidden transitions, such as dark exciton emission. ${ }^{9}$ At high $T$ instead coupling to acoustic phonons becomes important and the rapid thermalization makes thermal population of excited states the dominant factor in determining the band shape. ${ }^{9}$

In this work we investigate how the emission band shape of single CdSe nanocrystals changes as a function of the thermal population. We consider the most usual exciton complexes emitting in nanocrystals, namely, neutral excitons (X0), biexcitons $(\mathrm{XX})$, and singly charged excitons or trions $\left(\mathrm{X}^{ \pm}\right)$. Motivated by recent experiments, we study not only NDs but also elongated nanocrystals, or nanorods (NRs). Photoluminescence measurements showed that the band shape of NRs displays a distinct $T$ dependence as compared to that of NDs or tetrapods. ${ }^{10}$ Our calculations reveal that the occupation of excited states in typical NDs is moderate even at room $T$, but it becomes critical in NRs due to the weak longitudinal confinement, which implies large density of states (DOS). This makes the emission spectrum of NRs particularly sensitive to the $T$. Contrary to the bulk, where simple band shapes follow from the Boltzmann occupation of the continuum of states, the shapes in nanocrystals can be quite complex. This is because the spatial confinement im- poses different DOS and orbital symmetries to the initial and final states. These factors differ for each kind of excitonic complex so that characteristic shapes are observed for these species.

\section{THEORETICAL METHOD}

The theoretical model we employ is an effective-mass configuration-interaction (CI) scheme, which we have previously used to describe the zero- $T$ emission of NRs. ${ }^{11}$ The single-particle states are obtained from three-dimensional single-band Hamiltonians for conduction electrons, heavy holes (HH), and light holes (LH). ${ }^{12}$ The exciton or multiexciton states are then computed with a full CI method on a basis of Hartree products of two Slater determinants of spin orbitals, one for the $N_{e}$ electrons and the other one for the $N_{h}$ holes of the $\left(N_{e}, N_{h}\right)$ excitonic complex. Note that the choice of a CI method is demanded by the importance of Coulomb correlations to estimate the emission properties of NDs (Ref. 9) and NRs, ${ }^{13,14}$ along with the necessity of properly describing excited states. ${ }^{15}$ The emission probability from an initial state $|i\rangle$ is calculated within the dipole approximation and Fermi's golden rule as

$$
\tau_{i}^{-1}(\omega)=\sum_{f}\left(|\langle f|\hat{\mathcal{P}}| i\rangle|^{2} \Gamma\left(E^{i}-E^{f}-\hbar \omega\right) \quad p_{i}(T),\right.
$$

where $|f\rangle$ is the final state, $\hat{\mathcal{P}}$ is the polarization operator, ${ }^{11} E^{s}$ is the energy of the state $|s\rangle$, and $\Gamma(E)$ is a Lorentzian curve centered at energy $E$, which simulates the intrinsic bandwidth of the transition. Since we consider a large number of possible transitions, their resonances will overlap to yield a broader band. We assume the Boltzmann distribution $p_{i}(T)=\frac{g_{i}}{g_{0}} e^{-\Delta E_{i} / k T}$ for the initial-state occupation at temperature $T$, with $g_{i}\left(g_{0}\right)$ as the degeneracy factor of the state $|i\rangle$ (ground state), $\Delta E_{i}$ the energy difference between $|i\rangle$ and the ground state, and $k$ the Boltzmann constant. We then use an intermediate normalization, i.e., the ground-state occupation is always set to one.

We study one spherical ND and two elongated NRs with different aspect ratio. The ND is modeled as a sphere of radius $R=4 \mathrm{~nm}$ while the NRs are modeled as cylinders of length $L(L=5$ and $L=20 \mathrm{~nm})$ with hemispherical caps of radius $R=2 \mathrm{~nm}$ attached to the ends [see geometry in Fig. $1(\mathrm{a})$. Note that the volume of the ND and that of the larger NR are comparable. The nanocrystals we consider are free 

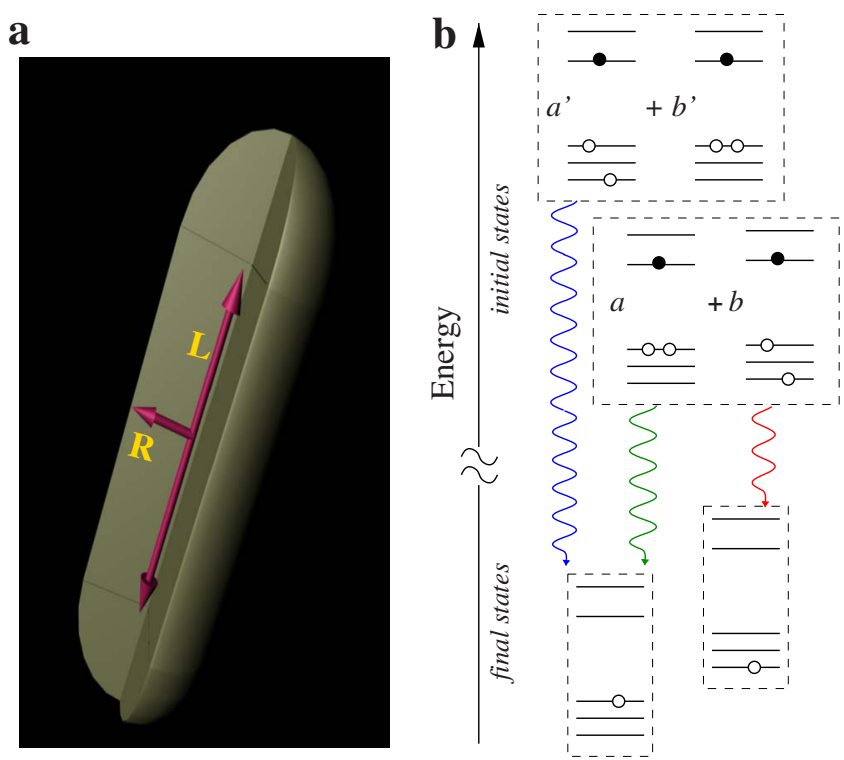

FIG. 1. (Color online) (a) Section of the nanostructure geometry under study. (b) Diagrammatic representation of a few transitions between initial $\mathrm{X}^{+}$states and final hole states. The two dominant configurations of the $\mathrm{CI}$ expansion are shown for the $\mathrm{X}^{+}$states with solid and empty dots standing for electrons and holes, respectively (see text).

from surface states, which allows us to focus on the influence of the (volume) energy structure exclusively. In practice, such samples can be obtained by properly growing semiconductor shell layers or surface bound ligands over the core. We use the same material parameters and confining potentials as in Ref. 11. The full CI basis set is built from the 16 lowest-electron $(\mathrm{HH}, \mathrm{LH})$ spin orbitals with envelope angular momentum $m_{z}=0$ and the four lowest with $m_{z}= \pm 1$. The resulting dimension of the resulting Hilbert spaces for $\mathrm{X} 0$, $\mathrm{X}^{-}, \mathrm{X}^{+}$, and $\mathrm{XX}$, is $400,3800,3800$, and 36100 , respectively. We calculate all possible optical transitions from the 50 lowest states of the initial excitonic complex to the six lowest states of the final complex. We have checked that adding more states does not significantly alter the emission spectra. ${ }^{16}$ A Lorentzian bandwidth of $0.5 \mathrm{meV}$ is taken, a standard low- $T$ homogeneous bandwidth, which allows us to resolve the fine details of the spectra. At high $T$ the homogeneous bandwidth increases due to electron-phonon interaction. Thus, for room $T$ we also show results with bandwidth $10 \mathrm{meV}$.

\section{RESULTS AND DISCUSSION}

In order to better understand the emission spectra, it is worth analyzing a simplified case first. In Fig. 1(b) we illustrate a few transitions corresponding to the recombination of $\mathrm{X}^{+}$. Two initial trion states are shown, each with its two dominant configurations $\left(a>b\right.$ and $\left.a^{\prime}>b\right)$. Solid (empty) dots are used to represent electrons (holes) in their singleparticle orbitals. At zero temperature only the lowest trion state is initially occupied. From an inspection of the dominant configuration $(a)$, one can see that electron-hole recom- bination leads to a hole in the ground state (middle arrow in green). The energy of the transition is given by the energy difference between the trion and hole states. There are however additional transitions enabled by the Coulomb mixing. Thus, the second dominant configuration $(b)$ opens a channel for recombination yielding a hole in an excited orbital (right arrow in red). This transition is redshifted with respect to the fundamental one. As $T$ increases, higher trion states become occupied. These may also recombine to form single hole states. In the example of Fig. 1(b), the excited trion state undergoes a transition to the hole ground state (left arrow in blue), which is blueshifted with respect to the fundamental one. In general, multiple transitions arise from each initial state (especially when Coulomb mixing is strong). The ground state of the initial species gives the fundamental transition and possibly a few (smaller) redshifted ones. The excited states in turn may give either redshifted or blueshifted transitions, depending on the energy difference between the initial and final state.

In Fig. 2 we plot the lowest-lying energy levels of the different species we study for a NR of $L=5 \mathrm{~nm}$ (upper panels) and $L=20 \mathrm{~nm}$ (lower panels). Clearly, the density of states increases rapidly with decreasing longitudinal confinement. Besides, it is clear that the density of states increases with the complexity of the species (compare e.g., the $\mathrm{X} 0$ and XX panels). With growing $T$, a larger number of initial states are thermally occupied. This dramatically increases the number of possible transitions for complex excitonic species $\left(\mathrm{X}^{+}, \mathrm{X}^{-}, \mathrm{XX}\right)$. A detailed analysis of all these transitions is beyond the scope of this paper. Instead, we will compute them and derive some general trends as a function of $T$.

We start by inspecting the emission spectrum of X0. Figure 3 illustrates the spectra in the ND [Fig. 3(a)], NR with $L=5 \mathrm{~nm}$ [Fig. 3(b)] and NR with $L=20 \mathrm{~nm}$ [Fig. 3(c)] at different $T$. In the ND, at low $T(T=4 \mathrm{~K})$ only the X0 ground state is appreciably populated. As a result, only the fundamental transition is visible. With increasing $T$, a second resonance shows up at higher energies, coming from the exciton with a hole in the lowest LH orbital. Similar pictures arise for the NRs, Figs. 3(b) and 3(c), except that now the fundamental transition involves the $\mathrm{LH}$ instead of the $\mathrm{HH}$. This is due to the HH-LH ground-state crossover as the $\mathrm{NC}$ is elongated. ${ }^{11,17}$ All in all the $\mathrm{X} 0$ spectrum is quite clean, not only for NDs, ${ }^{9}$ as previously noted by Califano et al., ${ }^{9}$ but also for NRs. The contribution of the optically active excited states is small because they are beyond the thermal energy even at room $T$. Whereas for NDs this is just a single-particle effect, for NRs it is a result of the strong Coulomb correlations. Actually, in a single-particle description, the excited X0 states with significant emission intensity would be much closer to the ground state and hence visible at room $T$. This is illustrated by the dotted lines in Fig. 5(c), which show the single-particle spectrum at room $T$.

The dashed line at room $T$ shows the envelope emission band which results from using a bandwidth of $10 \mathrm{meV}$. The broader bandwidth smears out the fine spectrum details and yields a coarse band whose shape should be comparable with that observed in high- $T$ experiments. As can be seen in Fig. 3 , in general the envelope emission band differs from the simple Boltzmann distributions of bulk semiconductors ${ }^{18}$ as 

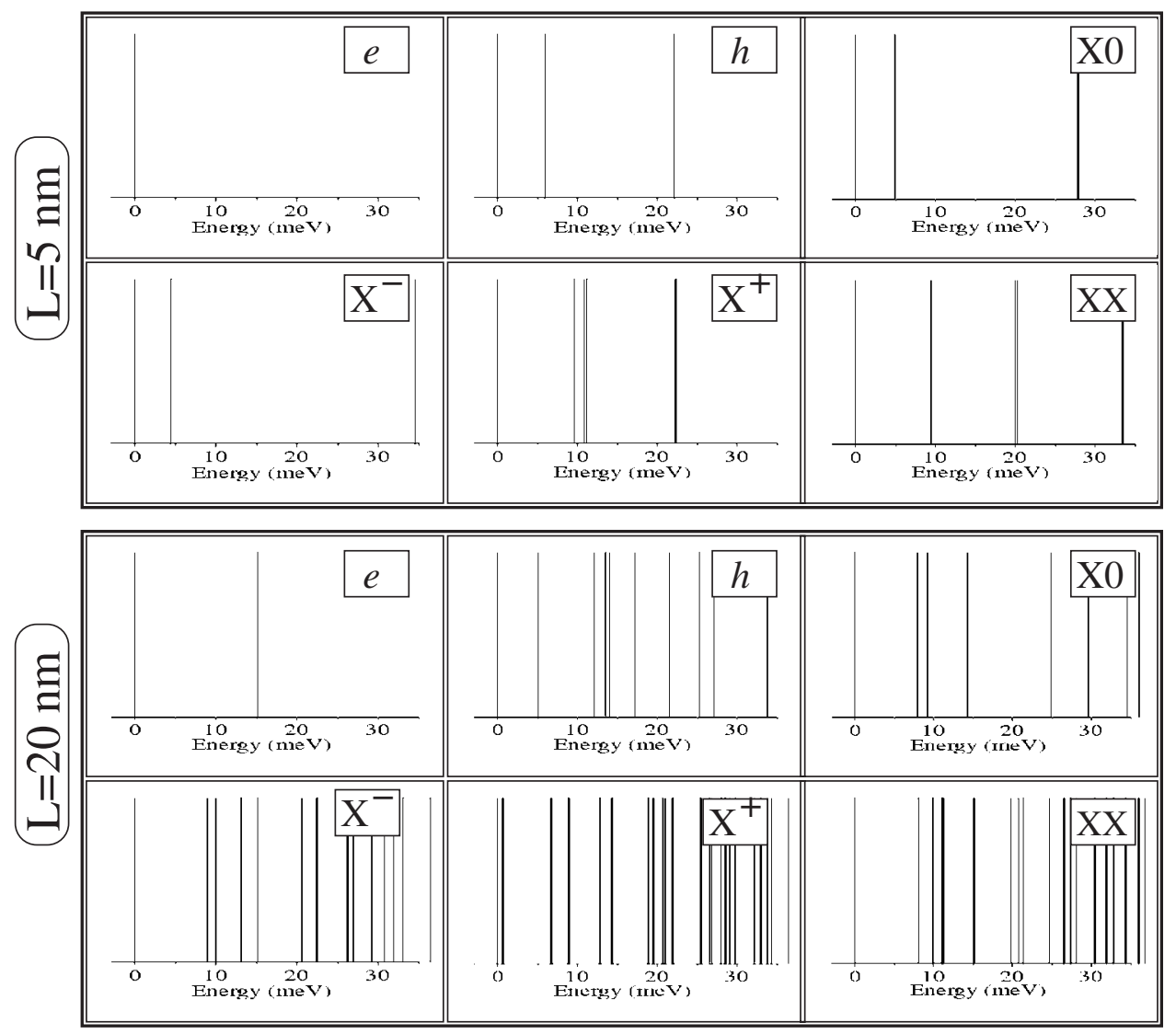

FIG. 2. Density of states for electron $(e)$, hole $(h)$, and different excitonic complexes $\left(\mathrm{X} 0, \mathrm{X}^{-}, \mathrm{X}^{+}, \mathrm{XX}\right)$ in a $\mathrm{NR}$ with $L=5 \mathrm{~nm}($ top panels) and a NR with $L=20 \mathrm{~nm}$ (bottom panels). The first line in each panel corresponds to the ground state.

the shape is now determined by the discrete DOS of the ND. The emission decays exponentially on the left side of the fundamental transition but it deviates from the exponential behavior on the right side due to influence of the excited resonances. Yet, for $\mathrm{X} 0$ this deviation is small because there are very few excited resonances. The emission band maximum is near the fundamental transition energy except in

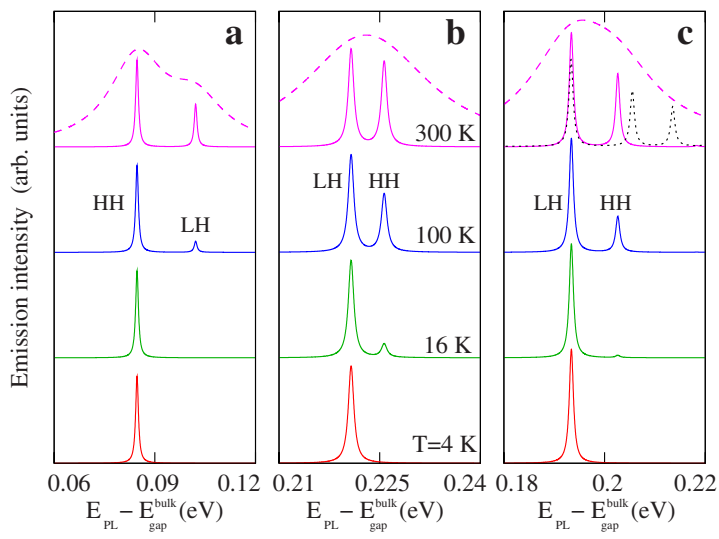

FIG. 3. (Color online) Exciton emission spectrum as a function of the $T$ for (a) a spherical ND, (b) a NR with $L=5 \mathrm{~nm}$, and (c) a NR with $L=20 \mathrm{~nm}$. The dashed line at $T=300 \mathrm{~K}$ shows the shape of the emission band assuming broad bandwidth. The dotted line in (c) shows the emission neglecting Coulomb interaction (the spectrum is shifted to match the energy scale of the interacting one). short NRs, where the lowest $\mathrm{HH}$ and LH orbitals are close in energy. In such a case, since the corresponding resonances have similar intensities, the maximum is centered in between them [see Fig. 3(b)].

It is worth noting that transitions showing up at lower (higher) energy than the fundamental one involve excited final (initial) states. Since the final state of the X0 transitions is the vacuum, there can be no resonances below the fundamental one, and the band always decays exponentially on the left side.

Recent experiments showed that the exciton band shape of CdSe NRs displays a more complex $T$ dependence than NDs. ${ }^{10}$ In particular, NRs with average diameter $3.2 \mathrm{~nm}$ and total length $13.4 \mathrm{~nm}$ exhibited a broadening mechanism with an activation energy of $4.2 \mathrm{meV}$. This was tentatively ascribed to possible fluctuating surface charges on the NR surface or to the dark-to-bright exciton energy splitting. From our study, this can also be ascribed to the thermal population of the low-energy $\mathrm{HH}$ or $\mathrm{LH}$ states. Indeed, for rods of total length $L=14 \mathrm{~nm}$ we predict the energy splitting between the ground state (X0 with $\mathrm{LH}$ ) and the first excited state (X0 with $\mathrm{HH}$ ) to be $7.5 \mathrm{meV}$, in the order of the experimental value. As a result from the activation of the $\mathrm{HH}$ resonance, the bandwidth of the NR spectrum in Fig. 3(b) saturates around $T=100 \mathrm{~K}$, when $\mathrm{HH}$ and $\mathrm{LH}$ states reach comparable populations. By contrast, in the ND spectrum of Fig. 3(a), the excited resonance keeps on increasing up to $300 \mathrm{~K}$, further modifying the bandwidth. This is consistent with the behavior reported in Fig. 3 of Ref. 10 


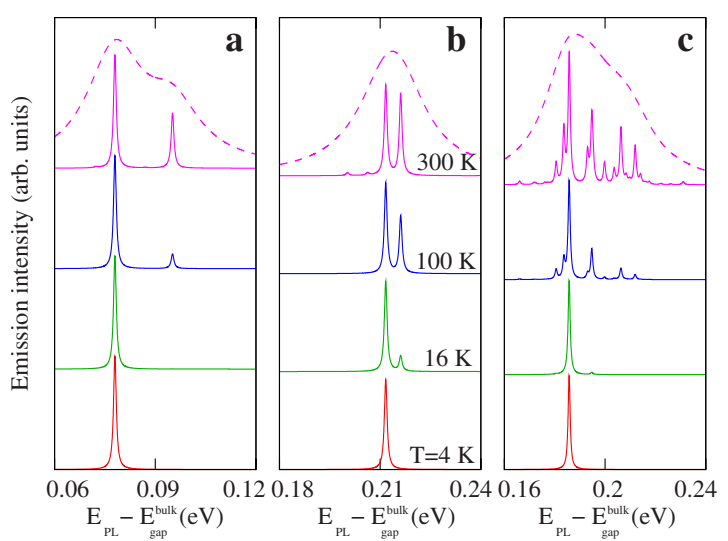

FIG. 4. (Color online) Same as Fig. 3 but for a negatively charged exciton.

Next we analyze the $\mathrm{X}^{-}$spectrum. A major difference with respect to $\mathrm{X} 0$ is that there are now many possible final states, which correspond to the single electron. The $\mathrm{X}^{-}$spectra are illustrated in Fig. 4. One can see that the ND results are similar to those of X0. The NR spectra, however, are much richer. They show a large number of resonances at high $T$ (see $T \geq 100 \mathrm{~K}$ ). Most of them show up at higher energies than the fundamental transition (the only visible one at low $T$ ) but a few show up at lower energies too. The high-energy (low-energy) resonances mainly involve excited $\mathrm{X}^{-}$(electron) states. The fact that the former are more abundant than the latter is because the DOS of $\mathrm{X}^{-}$is larger than that of single electrons. As a consequence of the DOS, the emission band of $\mathrm{X}^{-}$decays almost exponentially on the low-energy side but it has a slower, irregular decay on the high-energy side.

The $\mathrm{X}^{+}$spectrum is plotted in Fig. 5. Again, there are now multiple possible initial and final states. Yet, the final states correspond to the single hole, which is heavier than the electron and hence possesses a denser DOS. It then turns out that the $\mathrm{X}^{+}$spectrum has several resonances at energies below the fundamental transition. This can be already observed for the spherical ND [Fig. 5(a) at $T \geq 100 \mathrm{~K}$ ]. The presence of these

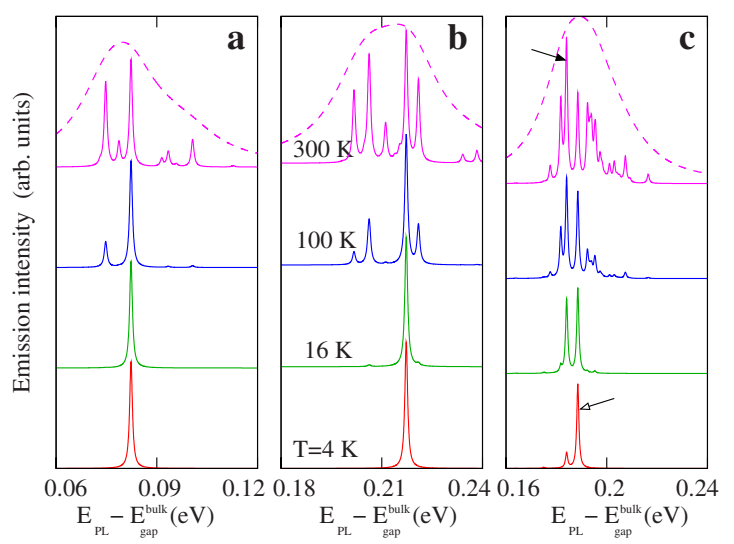

FIG. 5. (Color online) Same as Fig. 3 but for a positively charged exciton. The arrows in (c) point at the most intense resonance at low and high $T$, which correspond to different optical transitions.

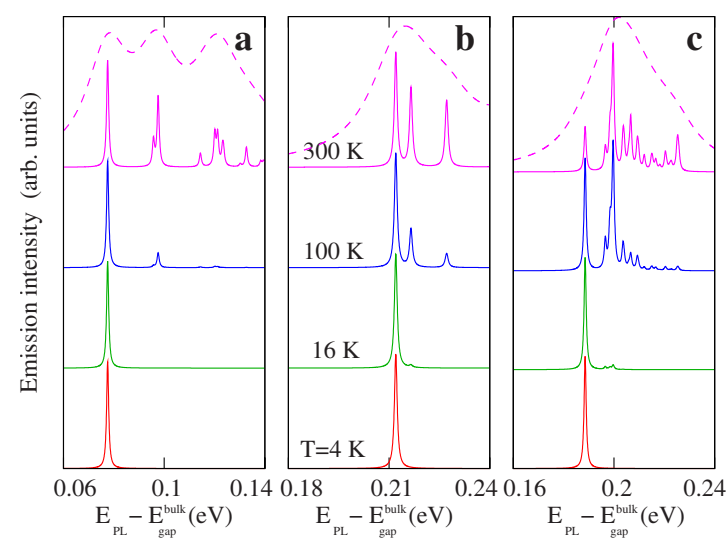

FIG. 6. (Color online) Same as Fig. 3 but for a biexciton.

resonances implies that the decay of the emission band on the low-energy side is no longer well described by a simple exponential.

Interestingly, at room $T$, some of initially weak resonances may become more intense than the fundamental transition one. ${ }^{19}$ Compare, e.g., in Fig. 5(c) the fundamental transition (empty arrow) with that indicated by the solid arrow. These intense resonances produce a few meV shift of the emission band maximum with respect to the fundamental transition energy.

Last, we investigate the XX spectrum in Fig. 6. In this case several high-energy resonances appear as the $T$ increases, owing to the large DOS of XX. Surprisingly, however, no resonance appears below the fundamental transition one. This is because the XX ground state has a dominant closed shell electronic configuration with two electrons in the lowest conduction orbital and two holes in the lowest valence orbital. Since the dipole matrix element is only large when the initial and final states have significant orbital overlap, ${ }^{11}$ only the $\mathrm{X} 0$ ground state gives sizable coupling. Excited X0 states, which should enable the low-energy transitions, have electrons or holes in orbitals with different symmetries to those occupied by the XX ground state, so the dipole coupling is severely suppressed.

As in the case of $\mathrm{X}^{+}$, the large DOS of $\mathrm{XX}$ makes it possible to obtain high-energy resonances that are stronger than the fundamental transition [see, e.g., Fig. 6(c)]. Moreover, since there are many high-energy transitions and no low-energy one to compensate, the emission band of XX can be significantly blueshifted with respect to the fundamental transition energy. In Fig. 6(c), at room $T$, the maximum of the emission band (dashed line) is $14 \mathrm{meV}$ above the fundamental transition. We finally mention that due to the absence of low-energy transitions, the emission band shape of XX clearly differs from the inverse Boltzmann distribution of higher-dimensional nanostructures,${ }^{20}$ which has been eventually applied to the study of NRs. ${ }^{14}$

\section{CONCLUSIONS}

In conclusion, we have presented effective-mass CI simulations of the emission spectra of $\mathrm{X} 0, \mathrm{X}^{+}, \mathrm{X}^{-}$, and $\mathrm{XX}$ in CdSe NDs and NRs at finite temperatures. Thermal popula- 
tion of the discrete excited states changes the shape of the emission bands. These effects are particularly pronounced in NRs, due to the large density of states, and for complex excitonic species (trions and biexcitons), due to the different nature of the initial and final states. For X0 and XX thermal population modifies the high-energy side of the emission band while for trions it modifies both low- and high-energy sides. The characteristic band shape of each excitonic species should be useful for single-dot spectral assignments. For example, we have shown that the presence of multiple resonances below the fundamental transition is characteristic of $\mathrm{X}^{+}$. This strongly supports the assignment proposed in recent photoluminescence experiments. ${ }^{21}$ The different $T$ dependence we observe for NDs and NRs is also consistent with Ref. 10 experiments.

\section{ACKNOWLEDGMENTS}

The CI calculations were performed using A. Bertoni's CITOOL software code. Support from MCINN under Project No. CTQ2008-03344, UJI-Bancaixa under Project No. P11B2006-03 and the Ramon y Cajal program (JIC) is acknowledged. *josep.planelles@qfa.uji.es

${ }^{1}$ U. Woggon, J. Appl. Phys. 101, 081727 (2007).

${ }^{2}$ P. Reiss, J. Bleuse, and A. Pron, Nano Lett. 2, 781 (2002).

${ }^{3}$ M. Nirmal and L. Brus, Acc. Chem. Res. 32, 407 (1999).

${ }^{4}$ D. E. Gómez, M. Califano, and P. Mulvaney, Phys. Chem. Chem. Phys. 8, 4989 (2006).

${ }^{5}$ D. Valerini, A. Creti, M. Lomascolo, L. Manna, R. Cingolani, and M. Anni, Phys. Rev. B 71, 235409 (2005).

${ }^{6}$ T. J. Liptay and R. J. Ram, Appl. Phys. Lett. 89, 223132 (2006).

${ }^{7}$ A. Joshi, K. Y. Narsingi, M. O. Manasreh, E. A. Davis, and B. D. Weaver, Appl. Phys. Lett. 89, 131907 (2006).

${ }^{8}$ Q. Dai, Y. Song, D. Li, H. Chen, S. Kan, B. Zou, Y. Wang, Y. Deng, Y. Hou, S. Yu, L. Chen, B. Liu, and G. Zou, Chem. Phys. Lett. 439, 65 (2007).

${ }^{9}$ M. Califano, A. Franceschetti, and A. Zunger, Nano Lett. 5, 2360 (2005).

${ }^{10}$ A. Al Salman, A. Tortschanoff, M. B. Mohamed, D. Tonti, F. van Mourik, and M. Chergui, Appl. Phys. Lett. 90, 093104 (2007).

${ }^{11}$ F. Rajadell, J. I. Climente, J. Planelles, and A. Bertoni, J. Phys. Chem. C 113, 11268 (2009).

${ }^{12} \mathrm{HH}$ and $\mathrm{LH}$ are decoupled in our model. Their presumably weak coupling due to spin-orbit interaction would barely affect the density states and hence the main spectral features we describe.

${ }^{13}$ J. I. Climente, M. Royo, J. L. Movilla, and J. Planelles, Phys. Rev. B 79, 161301(R) (2009).

${ }^{14}$ S. Baskoutas and A. F. Terzis, J. Appl. Phys. 98, 044309 (2005).

${ }^{15}$ We neglect electron-hole exchange, which splits bright- and dark-exciton states, because in the high- $T$ regime both kinds of state have similar populations.

${ }^{16}$ Six final states suffice to describe the spectrum because the dominant electronic configurations of the initial states involve low-energy orbitals only.

${ }^{17}$ P. C. Sercel and K. J. Vahala, Phys. Rev. B 44, 5681 (1991).

${ }^{18} \mathrm{P}$. Yu and M. Cardona, Fundamentals of Semiconductors (Springer, Berlin, 1996).

${ }^{19}$ The origin of such resonances is not a single transition but rather the sum of many closely packed transitions.

${ }^{20}$ B. P. Zhang, N. T. Binh, Y. Segawa, Y. Kashiwaba, and K. Haga, Appl. Phys. Lett. 84, 586 (2004).

${ }^{21}$ X. Wang, X. Ren, K. Kahen, M. A. Hahn, M. Rajeswaran, S. Maccagnano-Zacher, J. Silcox, G. E. Cragg, A. L. Efros, and T. D. Krauss, Nature (London) 459, 686 (2009). 